\title{
CONTEXTUAL EQUIVALENCE FOR HIGHER-ORDER $\pi$-CALCULUS REVISITED
}

\author{
ALAN JEFFREY $^{a}$ AND JULIAN RATHKE $^{b}$ \\ ${ }^{a}$ Bell Labs, Lucent Technologies, and CTI, DePaul University \\ e-mail address: ajeffrey@bell-labs.com \\ ${ }^{b}$ School of Informatics, University of Sussex \\ e-mail address: julianr@sussex.ac.uk
}

\begin{abstract}
The higher-order $\pi$-calculus is an extension of the $\pi$-calculus to allow communication of abstractions of processes rather than names alone. It has been studied intensively by Sangiorgi in his thesis where a characterisation of a contextual equivalence for higher-order $\pi$-calculus is provided using labelled transition systems and normal bisimulations. Unfortunately the proof technique used there requires a restriction of the language to only allow finite types.

We revisit this calculus and offer an alternative presentation of the labelled transition system and a novel proof technique which allows us to provide a fully abstract characterisation of contextual equivalence using labelled transitions and bisimulations for higher-order $\pi$-calculus with recursive types also.
\end{abstract}

\section{INTRODUCTION}

It is evident that there is growing interest in the study of mobile code in process languages [3, 1, 9, 15]. It is also clear that there is some relationship between the use of higher-order features and mobility. Indeed, code mobility can be expressed as communication of process abstractions. For this reason then it is important for us to develop a clear understanding of the use of higher-order features in process languages.

Work towards this began several years ago with various proposals for higher-order versions of known calculi [14 4], including the higher-order $\pi$-calculus or $\mathrm{HO} \pi$ [10]. This calculus was studied intensively by Sangiorgi and one of his achievements was to provide a translation of the higherorder language which supports code mobility, to a first-order $\pi$-calculus which supports only name mobility. This translation is proved to be fully abstract with respect to barbed congruence, but with the restriction to a language of finite types.

While the translation is of interest in its own right, it also turned out to be very useful for providing a powerful fully abstract characterisation of barbed congruence in terms of labelled transition systems and normal bisimulations. Providing direct proof techniques for contextual equivalences in higher-order process languages is often considered to be hard [13]. In this paper, the difficulty

2000 ACM Subject Classification: D.3.1.

Key words and phrases: Higher-order languages, concurrency, full abstraction.

${ }^{a}$ This material is based upon work supported by the National Science Foundation under Grant No. 0430175.

${ }^{b}$ Research partially funded by the Nuffield Foundation.

LOGICAL METHODS
DOI:10.2168/LMCS-1 (1:4) 2005
(C) A. Jeffrey and J. Rathke

(c) Creative Commons 
arises in establishing soundness of the proof technique, which is tantamount to establishing some sort of contextuality property. It has been seen that the use of a translation of higher- to first-order communication can alleviate this problem and such translations have been employed to this effect [11 7].

However, due to the restriction to finite types for the correctness of these translations, the soundness of the proof technique is only guaranteed for finite types. Given that recursive types are used extensively in $\pi$-calculus, for encodings of datatypes and functions, this poses a significant restriction. Sangiorgi has shown that by studying various subcalculi, such as the asynchronous $\pi$ calculus, he is able to remove the restriction to finite types [13]. To date, there has been no proof of full abstraction for full $\mathrm{HO} \pi$ in the presence of recursive types.

In this paper we present an alternative description of labelled transition systems and normal bisimulations for $\mathrm{HO} \pi$, which is informed by Sangiorgi's translation of higher-order to first-order communication. Our alternative presentation allows a direct proof of soundness for contextual equivalence which makes no use of the translation to first-order $\pi$-calculus and, more importantly, makes no restriction on types.

The innovation here lies in the introduction of operators $\tau_{k}$ and $\langle k \Leftarrow v\rangle$ which simulate the triggers $\operatorname{Tr}_{k}$ and meta-notation $\{k:=v\}$ of Sangiorgi [11] where $k$ is a unique identifier for the trigger and $v$ is a process abstraction. The crucial difference is that where Sangiorgi gives definitions as $\mathrm{HO} \pi$ terms for these devices:

$$
\operatorname{Tr}_{k}=(x) k\langle x\rangle \quad \text { and } \quad\{k:=v\}=* k(x) v \cdot x
$$

where $k\langle x\rangle$ represents an output on name $k$ and $* k(x) P$ represents a replicated input on name $k$, we leave the operators uninterpreted. There are no interactions between the operators $\tau_{k}$ and $\langle k \Leftarrow v\rangle$. Rather, we just mimic the behaviour of triggers in the labelled transition systems. The benefit of doing this is that it allows us to obtain a direct soundness proof that (normal) bisimilarity implies contextual equivalence without recourse to any translation in its correctness proof.

A challenge of approaching the problem in this way is that it is not immediately clear that bisimilarity will be complete for contextual equivalence in $\mathrm{HO} \pi$. That is to say, it is not obvious whether each transition has a genuine $\mathrm{HO} \pi$ context which validates it. At this point however we can interpret the operators $\tau_{k}$ and $\langle k \Leftarrow v\rangle$ as HO $\pi$ terms exactly as Sangiorgi does. It is then a simple matter to demonstrate completeness following familiar techniques [3, 7] 5]. The real payoff is that not only do we obtain a direct soundness proof but the postponement of interpreting the triggers allows us to finesse any restrictions to finite types.

The remainder of the paper is organised as follows: in Section 2 we recall the syntax and semantics of $\mathrm{HO} \pi$ along with the definition of contextual equivalence which we will be using. This is followed in Section 3 by a presentation of the novel labelled transition system using the operators $\tau_{k}$ and $\langle k \Leftarrow v\rangle$. We prove that bisimilarity over this labelled transition system is sound for contextual equivalence in Section 4 and conversely, that it is complete for contextual equivalence in Section 5. We conclude in Section 6 with some closing remarks.

\section{HIGHER-ORDER $\pi$ CALCULUS}

Except for small changes in notation the language is as can be found in [13] with three main differences:

(1) We assume two distinct countably infinite sets of identifiers, $\mathcal{V}$ and $\mathcal{N}$, for variables and channel names respectively. In general we will use $x, y, z$ to range over variables and $a, b, c$ to range over channel names. This variable/name distinction makes the algebraic properties 


\begin{tabular}{|c|c|c|c|}
\hline \multirow{6}{*}{\multicolumn{2}{|c|}{$T, U$}} & & Value Types \\
\hline & & $\cdot$ & Unit type \\
\hline & & $\operatorname{ch}[T]$ & Channel type \\
\hline & & $T \rightarrow \diamond$ & Abstraction type \\
\hline & & $Z$ & Type variable \\
\hline & & $\operatorname{rec} Z . T$ & Recursive type \\
\hline \multirow[t]{9}{*}{$P, Q$} & $::=$ & & Terms \\
\hline & & $v \cdot w$ & Application \\
\hline & & $v(x: T) P$ & Input \\
\hline & & $v\langle w\rangle P$ & Output \\
\hline & & if $v=w$ then $P$ else $Q$ & Matching \\
\hline & & $\vee(a: T) \cdot(P)$ & Name creation \\
\hline & & $P \| Q$ & Concurrency \\
\hline & & $* P$ & Repetition \\
\hline & & $\mathbf{0}$ & Termination \\
\hline \multirow[t]{5}{*}{$v, w$} & $::=$ & & Values \\
\hline & & $\cdot$ & Unit value \\
\hline & & $a$ & Channel name \\
\hline & & $x$ & Variable \\
\hline & & $(x: T) P$ & Abstractions \\
\hline
\end{tabular}

Figure 1: The Syntax

of the language a little cleaner and we are confident that the techniques proposed here would also be applicable if we identified these sets.

(2) Since we have adopted a variable/name distinction, we have used Honda and Yoshida's definition of observational equivalence [6] in Section 2.4 rather than Sangiorgi's. See [2] for a discussion of this issue.

(3) We allow communication of channel names as well as process abstractions so that there is a core $\pi$-calculus as a direct subcalculus of $\mathrm{HO} \pi$.

2.1. Syntax. We present the syntax of $H O \pi$ in Figure 1 The grammar of types for values includes:

- $(\cdot)$ : a singleton type just containing the value $(\cdot)$.

- ch $[T]$ : the type of channels which can be used for communicating data of type $T$. Note that in this paper we are not considering input-only or output-only channels.

- $T \rightarrow \diamond:$ the type of an abstraction $(x: T) P$. Such an abstraction can be applied to a value $v$ of type $T$ to return a well-typed process $P[v / x]$.

- $Z$ and rec $Z$.T: these allow recursive types, such as the type for monomorphic $\pi$-calculus channels rec $Z$.ch $[Z]$. We require $Z$ to be guarded: any free occurrence of $Z$ lies within a subexpression of $T$ of the form $\operatorname{ch}[U]$ or $U \rightarrow \diamond$.

The grammar of process terms includes: 
- $v \cdot w$ : the application of abstraction $v$ to argument $w$. During execution, $v$ will be instantiated by an abstraction of the form $(x: T) P$, and $\beta$-reduction will give the process $P[w / x]$.

- $v(x: T) P$ and $v\langle w\rangle P$, which are the standard synchronous input and output of the $\pi$-calculus, except that since abstractions are first-class values, we can communicate higher-order data as well as first-order data.

- if $v=w$ then $P$ else $Q$ : an equality test on values, where the type system will ensure that $v$ and $w$ are channels, and so we will never compare abstractions for syntactic identity.

- $v(a: T) .(P), P \| Q, * P$ and $\mathbf{0}$ : the standard $\pi$-calculus processes for channel generation, concurrency, replication and termination.

The grammar of values includes:

- $(\cdot)$ : the only value of type $(\cdot)$.

- $a$ and $x$ : channel names and variables respectively.

- $(x: T) P$ : an abstraction, which can be applied to a value $v$ to return a process $P[v / x]$. Since abstractions are considered first-class values, they can be communicated on channels, or passed as arguments to other abstractions. This feature gives $\mathrm{HO} \pi$ its higher-order power.

2.2. Reduction semantics. The reduction semantics for the language is defined in a standard manner: we first introduce the evaluation contexts

$$
\mathcal{E}::=[\cdot]|\mathcal{E} \| P| \text { va.E }
$$

Structural equivalence, $\equiv$ is defined to be the least congruence with respect to $\mathcal{E}$ contexts such that it makes $(\|, \mathbf{0})$ into a commutative monoid and moreover satisfies

$$
\begin{aligned}
\operatorname{va}(P \| Q) & \equiv v a \cdot P \| Q \quad \text { if } a \notin \mathrm{fn}(P) \\
* P & \equiv * P \| P
\end{aligned}
$$

We will now consider processes up to structural equivalence throughout the remainder. We define the reduction relation $\longrightarrow$ as the least precongruence with respect to $\mathcal{E}$ contexts such that the following axioms hold

$$
\begin{array}{llll}
(\text { comm }) & a\langle v\rangle P \| a(x) Q & \rightarrow P \|(x) Q \cdot v & \\
(\beta-\text { redn }) & (x) P \cdot v & \rightarrow P[v / x] \\
\text { (cond-tt) } & \text { if } a=a \text { then } P \text { else } Q & \rightarrow P & \\
\text { (cond-ff) } & \text { if } a=b \text { then } P \text { else } Q & \rightarrow Q
\end{array}
$$

In a standard notation we write $\Longrightarrow$ to denote the reflexive, transitive closure of $\longrightarrow$.

2.3. Type system. We introduce a simple type system for the language which comprises types for channels and abstractions, together with recursive types. To allow us to infer recursive types for terms we make use of type isomorphism. We define this by letting $\sim_{i s o}$ be the least congruence on types which includes

$$
\operatorname{rec} Z . T \sim_{\text {iso }} T[\operatorname{rec} Z . T / Z]
$$

A type environment $\Gamma$ is a finite set of mappings from identifiers (channel names or variables) to types with the restriction that channel names $a$ must be mapped to channel types of the form $\mathrm{ch}[T]$. We write $\Gamma, n: T$ to represent the environment made up of the disjoint union of $\Gamma$ and the mapping $n$ to $T$. We will call an environment closed if it contains mappings of channel names only and will 


$$
\begin{array}{ccc}
\frac{\Gamma(v)=T}{\Gamma \vdash v: T} & \frac{\Gamma, x: T \vdash P}{\Gamma \vdash(x: T) P: T \rightarrow \diamond} & \frac{\Gamma \vdash v: T \quad T \sim_{i s o} U}{\Gamma \vdash v: U} \\
\frac{\Gamma \vdash v: \operatorname{ch}[T], w: \operatorname{ch}[T]}{\Gamma \vdash P} \Gamma \vdash Q & & \frac{\Gamma \vdash P, Q}{\Gamma \vdash P \| Q, * P, \mathbf{0}} \\
\frac{\Gamma \vdash v: T: T \vdash P}{\Gamma \vdash v(a: T) \cdot(P)} & \frac{\Gamma \vdash P \quad \Gamma \vdash w: T \quad \Gamma \vdash v: \operatorname{ch}[T]}{\Gamma \vdash v\langle w\rangle P}
\end{array}
$$

Figure 2: The Typing Rules

write $\Delta$ to indicate this. Type inference rules for the calculus are given in Figure 2 We will call a well-typed process, $P$, closed if it can be typed as $\Delta \vdash P$ for some closed $\Delta$. It is easily shown that subject reduction holds for closed terms for the reduction relation and type inference system given.

2.4. Contextual equivalence. We will now define an appropriate notion of behavioural equivalence based on contexts and barbs.

Contexts are defined by extending the syntax of processes by allowing typed holes $[\cdot \Gamma]$ in terms. The type inference system is extended to contexts by using the rule

$$
\overline{\Gamma, \Gamma^{\prime} \vdash[\cdot \Gamma]}
$$

We write $C[]$ to denote contexts with at most one hole and $C[P]$ for the term which results from substituting $P$ into the hole.

For any given channel name $a$ such that $\Delta \vdash a: \operatorname{ch}[\cdot]$ we write $\Delta \models P \Downarrow a$ if there exists some $P^{\prime}, P^{\prime \prime}$ such that $P \Longrightarrow v \Delta^{\prime} .\left(a\langle\cdot\rangle P^{\prime \prime} \| P^{\prime}\right)$ with $a \notin \Delta^{\prime}$.

We use type-indexed families of relations $\left\{R_{\Delta}\right\}$ between closed process terms to describe equivalence. We will write $\mathcal{R}$ to refer to the whole family of relations and

$$
\Delta \models P R Q
$$

to indicate that $P$ and $Q$ are well-typed with respect to $\Delta$ and related by $R_{\Delta}$. For general process terms we define the open extension $\mathcal{R}^{o}$ of a typed relation $\mathcal{R}$ as

$$
\Delta, x_{1}: T_{1}, \ldots, x_{n}: T_{n} \models P \mathcal{R}^{o} Q
$$

holds if for every $\Delta^{\prime}$ disjoint from $\Delta$ and every $v_{i}$ such that $\Delta, \Delta^{\prime} \vdash v_{i}: T_{i}$ (for $1 \leq i \leq n$ ) we have

$$
\Delta, \Delta^{\prime} \models P\left[v_{1}, \ldots, v_{n} / x_{1}, \ldots, x_{n}\right] \mathcal{R} Q\left[v_{1}, \ldots, v_{n} / x_{1}, \ldots, x_{n}\right]
$$

Note that, in general, for closed terms $\Delta \models P R Q$ is not equivalent to $\Delta \models P \mathcal{R}^{o} Q$ as $\mathcal{R}^{o}$ enjoys the weakening property that $\Delta, \Delta^{\prime} \models P \mathcal{R}^{o} Q$ whenever $\Delta \models P \mathcal{R}^{o} Q$, even when $\mathcal{R}$ does not. However, the contextual equivalence which we study in this paper is defined as an open extension and therefore will satisfy this weakening.

There are a number of properties of type-indexed relations that we must define:

Symmetry:: A type-indexed relation $\mathcal{R}$ is symmetric whenever $\Delta \models P \mathcal{R} Q$ implies $\Delta \models Q \mathcal{R} P$. 
Reduction closure: A type-indexed relation $\mathcal{R}$ is reduction-closed whenever $\Delta \models P R Q$ and $P \longrightarrow P^{\prime}$ implies there exists some $Q^{\prime}$ such that $Q \Longrightarrow Q^{\prime}$ and $\Delta \models P^{\prime} \mathcal{R} Q^{\prime}$.

Contextuality:: A type-indexed relation $R$ is contextual whenever $\Gamma^{\prime} \models P R{ }^{o} Q$ and $\Gamma \vdash C\left[\cdot \Gamma^{\prime}\right]$ implies $\Gamma \models C[P] \mathcal{R}^{o} C[Q]$.

Barb preservation:: A type-indexed relation $\mathcal{R}$ is barb-preserving if $\Delta \models P R Q$ and $\Delta \models P \Downarrow a$ implies $\Delta \models Q \Downarrow a$.

Definition 2.1 (Contextual equivalence). Let $\cong$ be the open extension of the largest type-indexed relation which is symmetric, reduction-closed, contextual and barb-preserving.

For technical convenience it will be useful to work with a lighter definition of contextuality. We say that a relation $R$ is $\|$-contextual if it is preserved by all contexts of the form $[\cdot \Gamma] \| R$ and we let $\cong_{p}$ denote the open extension of the largest typed relation over processes which is symmetric, $\|$-contextual, reduction-closed and barb-preserving. The following lemma demonstrates that this lighter definition is sufficient.

Lemma 2.2 (Context lemma). $\Gamma \models P \cong Q$ if and only if $\Gamma \models P \cong{ }_{p} Q$

Proof. In Appendix A

\section{Full abStraction}

In this section, we will present a bisimulation equivalence for $\mathrm{HO} \pi$, and show that this equivalence is fully abstract for contextual equivalence.

3.1. Labelled transitions. We will use a labelled transition system to characterize $\cong$ over higherorder $\pi$-calculus terms. The style of the labelled transition system differs a little from previous transition systems offered for $\mathrm{HO} \pi$. Most notably, the nodes of the transition system are described using an augmented syntax rather than process terms alone. Specifically, for each $k$ drawn from a countable set of names disjoint from $\mathcal{N}$ and $\mathcal{V}$, we introduce two new operators:

$$
\tau_{k} \quad \text { and } \quad\langle k \Leftarrow v\rangle
$$

with the intuitive reading that $\tau_{k}$ is an indirect reference to an abstraction and $\langle k \Leftarrow v\rangle$ stores the abstraction to which $k$ refers so that access to $v$ is provided through interaction with $k$. The augmented syntax for nodes is given the grammar of configurations $C$ obtained by extending Figure 1 with:

$$
\begin{aligned}
v & :=\ldots \text { (as Figure 1) ... } \mid \tau_{k} \\
C & :=P|\langle k \Leftarrow v\rangle| v a: T .(C) \mid C \| C
\end{aligned}
$$

We impose a syntactic restriction on the augmented syntax so that in any configuration $C$ for any given $k$ then $\langle k \Leftarrow v\rangle$ appears at most once in $C$. Structural equivalence and reduction lift to $C$ in the obvious manner - note that there are no reduction rules given for $\tau_{k}$ and $\langle k \Leftarrow v\rangle$ though. We augment the type rules by considering judgements of the form

$$
\Gamma ; \Theta \vdash v: T \quad \text { and } \quad \Gamma ; \Theta \vdash C
$$

where $\Theta$ represents a set of mappings from reference names to types $T$. The rules in Figure 2 are easily decorated with the extra $\Theta$ environment. The further rules required are given by

$$
\frac{\Theta(k)=T}{\Gamma ; \Theta \vdash \tau_{k}: T \rightarrow \diamond} \quad \frac{\Theta(k)=T \quad \Gamma ; \Theta \vdash v: T \rightarrow \diamond}{\Gamma ; \Theta \vdash\langle k \Leftarrow v\rangle}
$$


Nodes of our labelled transition system then are well-typed closed terms of the augmented language of the form

$$
(\Delta ; \Theta \vdash C)
$$

The transitions are of the form $(\Delta ; \Theta \vdash C) \stackrel{\alpha}{\longrightarrow}(\Delta ; \Theta \vdash C)$ or $(\Delta ; \Theta \vdash C) \stackrel{\tau}{\longrightarrow}(\Delta ; \Theta \vdash C)$ where visible labels $\alpha$ are given by the grammar:

$$
\alpha::=v a . \alpha\left|v k . d\left\langle\tau_{k}\right\rangle !\right| v k . d\left\langle\tau_{k}\right\rangle ?|d\langle v\rangle ?| d\langle v\rangle !
$$

where write $d$ to mean either a channel name $a$ or an indirect reference name $k$. The transitions are presented in Figures 345 The intuition for these transitions is (eliding types for readability):

- $P \stackrel{a\langle v\rangle ?}{\longrightarrow} P^{\prime}$ : indicates that $P$ is prepared to input a value $v$ on channel $a$ and then perform as $P^{\prime}$. The type system enforces that $v$ is a first-order value, and not an abstraction. Moreover, in this case both $a$ and $v$ are pre-existing values, and were not generated fresh for this transition.

- $P \stackrel{k\langle\nu\rangle ?}{\longrightarrow} P^{\prime}$ : indicates that $P$ has provided a named abstraction reference $k$ to the environment, and that the environment is calling the abstraction with pre-existing argument $v$.

- $P \stackrel{v b . a\langle b\rangle ?}{\longrightarrow} P^{\prime}$ : indicates that $P$ is prepared to input a fresh channel $b$ on channel $a$ and then perform as $P^{\prime}$. This is the same as $P \stackrel{a\langle b\rangle}{\longrightarrow} P^{\prime}$, except that $b$ is now a fresh channel generated by the environment, and has not been seen before by the process.

- $P \stackrel{v b . k\langle b\rangle \text { ? }}{\longrightarrow} P^{\prime}$ : indicates that $P$ has provided a named abstraction reference $k$ to the environment, and that the environment is calling the abstraction with fresh argument $b$.

- $P \stackrel{v l . a\left\langle\tau_{l}\right\rangle \text { ? }}{\longrightarrow} P^{\prime}$ : indicates that $P$ is prepared to input an abstraction $l$ on channel $a$ and then perform as $P^{\prime}$. In this case, we do not record the abstraction itself in the label, but instead we just generate a fresh reference $l$ to the abstraction.

- $P \stackrel{v l . k\left\langle\tau_{l}\right\rangle \text { ? }}{\longrightarrow} P^{\prime}$ : indicates that $P$ has provided a named abstraction reference $k$ to the environment, and that the environment is calling that abstraction with argument $l$. In this case, $k$ must be a higher-order abstraction, so is expecting an abstraction as an argument. Rather than recording the abstraction itself in the label, we instead generate a fresh reference $l$ to the abstraction.

- Each of the above input transitions has a dual output transition, where the role of the process and environment are exchanged.

We write $\bar{\alpha}$ to denote the complement of an action $\alpha$, which is defined to be the action $\alpha$ with the input/output annotation inversed. We will often write $\Longrightarrow$ to mean the reflexive transitive closure of $\stackrel{\tau}{\longrightarrow}$ and $\stackrel{\alpha}{\Longrightarrow}$ to mean $\Longrightarrow \stackrel{\alpha}{\longrightarrow} \Longrightarrow$. The following proposition states that the labelled transition system is well-defined in the sense that the transition relation only relates well-typed terms.

Proposition 3.1. If $\Delta ; \Theta \vdash C$ and $(\Delta ; \Theta \vdash C) \stackrel{\alpha}{\longrightarrow}\left(\Delta, \Delta^{\prime} ; \Theta, \Theta^{\prime} \vdash C^{\prime}\right)$ then $\Delta, \Delta^{\prime} ; \Theta, \Theta^{\prime} \vdash C^{\prime}$ is a valid typing judgement.

Proof. Straightforward induction. 


$$
\begin{gathered}
\frac{C \longrightarrow C^{\prime}}{(\Delta ; \Theta \vdash C) \stackrel{\tau}{\longrightarrow}\left(\Delta ; \Theta \vdash C^{\prime}\right)} \quad \frac{(\Delta ; \Theta \vdash C) \stackrel{\alpha}{\longrightarrow}\left(\Delta^{\prime} ; \Theta^{\prime} \vdash C^{\prime}\right)}{(\Delta ; \Theta \vdash C \| D) \stackrel{\alpha}{\longrightarrow}\left(\Delta^{\prime} ; \Theta^{\prime} \vdash C^{\prime} \| D\right)} \\
\frac{(\Delta, a: T ; \Theta \vdash C) \stackrel{\alpha}{\longrightarrow}\left(\Delta, a: T, \Delta^{\prime} ; \Theta, \Theta^{\prime} \vdash C^{\prime}\right) \quad(a \notin \mathrm{fn}(\alpha))}{(\Delta ; \Theta \vdash v a: T . C) \stackrel{\alpha}{\longrightarrow}\left(\Delta, \Delta^{\prime} ; \Theta, \Theta^{\prime} \vdash v a: T . C^{\prime}\right)} \\
\frac{(\Delta, b: T ; \Theta \vdash C) \stackrel{d\langle b\rangle !}{\longrightarrow}\left(\Delta, b: T ; \Theta \vdash C^{\prime}\right) \quad(d \neq b)}{(\Delta ; \Theta \vdash v b: T . C) \stackrel{v b . d\langle b\rangle !}{\longrightarrow}\left(\Delta, b: T ; \Theta \vdash C^{\prime}\right)} \\
\frac{(\Delta, b: T ; \Theta \vdash C) \stackrel{d\langle b\rangle ?}{\longrightarrow}\left(\Delta, b: T ; \Theta \vdash C^{\prime}\right) \quad(d \neq b)}{(\Delta ; \Theta \vdash C) \stackrel{v b . d\langle b\rangle ?}{\longrightarrow}\left(\Delta, b: T ; \Theta \vdash C^{\prime}\right)}
\end{gathered}
$$

Figure 3: Structural labelled transition rules

3.2. Bisimilarity. We use a standard definition of (weak) bisimilarity to provide our characterisation of $\cong$ for $\mathrm{HO} \pi$ :

Definition 3.2. We call a symmetric relation, $R$, between nodes of the labelled transition system a bisimulation if whenever $(n, m) \in \mathcal{R}$ we have

- $n \stackrel{\tau}{\longrightarrow} n^{\prime}$ implies there exists some $m^{\prime}$ such that $m \Longrightarrow m^{\prime}$ and $\left(n^{\prime}, m^{\prime}\right) \in \mathcal{R}$

- $n \stackrel{\alpha}{\longrightarrow} n^{\prime}$ implies there exists some $m^{\prime}$ such that $m \stackrel{\alpha}{\Longrightarrow} m^{\prime}$ and $\left(n^{\prime}, m^{\prime}\right) \in \mathcal{R}$

Let bisimulation equivalence, or bisimilarity, $\approx$ be the largest bisimulation relation.

We will write

$$
\Delta ; \Theta \models C \approx D
$$

to mean that $\Delta ; \Theta \vdash C$ and $\Delta ; \Theta \vdash D$ are valid typing judgements and moreover, they are related by $\approx$ as nodes of the lts. In order to provide a bisimulation characterisation of $\cong$ over HO $\pi$ we will consider a subrelation of $\approx$ by restricting our attention to nodes of the form

$$
(\Delta ; \vdash P)
$$

whose terms are clearly definable in $\mathrm{HO}$. We will simply write (when $\Theta$ is empty)

$$
\Delta \models P \approx Q
$$

to indicate bisimilarity between such terms of $\mathrm{HO} \pi$ considered as nodes of the labelled transition system.

3.3. Soundness of bisimilarity for contextual equivalence. We need to demonstrate that bisimilarity implies contextual equivalence for all $\mathrm{HO} \pi$ processes. In particular, because of Lemma 2.2 we need only show that bisimilarity is contained in some symmetric, reduction-closed, barb preserving and $\|$-contextual relation. The key to achieving this is to study the $\|$-context closure of bisimilarity. If we can demonstrate that this is reduction-closed then we have our result. To do this we must establish a decomposition theorem for interactions. For instance, if $P$ and $Q$ are bisimilar and we compose each of them with a process $R$ then suppose

$$
P \| R \rightarrow S
$$




$$
\begin{gathered}
\frac{T \sim_{i s o} U \rightarrow \diamond}{(\Delta ; \Theta \vdash a(x: T) P) \stackrel{v k . a\left\langle\tau_{k}\right\rangle ?}{\longrightarrow}\left(\Delta ; \Theta, k: U \vdash(x: T) P \cdot \tau_{k}\right)} \\
\frac{\Theta(k) \sim_{i s o} T \rightarrow \diamond}{(\Delta ; \Theta \vdash\langle k \Leftarrow v\rangle) \stackrel{v l . k\left\langle\tau_{l}\right\rangle ?}{\longrightarrow}\left(\Delta ; \Theta, l: T \vdash v \cdot \tau_{l} \|\langle k \Leftarrow v\rangle\right)} \\
\frac{\Delta ; \Theta \vdash v: T \rightarrow \diamond}{(\Delta ; \Theta \vdash a\langle v\rangle P) \stackrel{v k . a\left\langle\tau_{k}\right\rangle !}{\longrightarrow}(\Delta ; \Theta, k: T \vdash\langle k \Leftarrow v\rangle \| P)} \\
\stackrel{\Theta(k)}{\stackrel{\left(\Delta ; \Theta \vdash \tau_{k} \cdot v\right) \stackrel{v l . k\left\langle\tau_{l}\right\rangle !}{\longrightarrow}}{\longrightarrow}(\Delta ; \Theta, l: T \vdash\langle l \Leftarrow v\rangle)}
\end{gathered}
$$

Figure 4: Basic higher-order labelled transition rules

represents an interaction between $P$ and $R$. We decompose this into complementary actions

$$
P \stackrel{\alpha}{\longrightarrow} P^{\prime} \quad \text { and } \quad R \stackrel{\bar{\alpha}}{\longrightarrow} R^{\prime}
$$

respectively. Note however that $S$ is not necessarily obtained by a parallel composition of the targets of the transitions: $P^{\prime} \| R^{\prime}$. Instead, $P^{\prime}$ and $R^{\prime}$ may contain indirect references and their corresponding resources. These need to be matched up correctly to obtain $S$. We achieve this by introducing the merge (partial) operator $\langle\langle\cdot\rangle\rangle$ which will match up these terms and replace every indirect reference to an abstraction with the abstraction itself. We write

$$
C\left[v / \tau_{k}\right]
$$

to denote the substitution of the value $v$ for every instance of the indirect reference $\tau_{k}$. We define $\langle\langle C\rangle\rangle$ then as the operator on terms of the augmented syntax (up to $\equiv$ ) such that

$$
\begin{aligned}
\langle\langle C\rangle\rangle & =C & & \text { if } C \text { doesn't contain }\langle k \Leftarrow v\rangle \text { for any } k, v \\
\langle\langle v(\vec{a}: \vec{T}) \cdot(\langle k \Leftarrow v\rangle \| C)\rangle\rangle & =\left\langle\left\langle v(\vec{a}: \vec{T}) .\left(C\left[v / \tau_{k}\right]\right)\right\rangle\right\rangle & & \text { if } \tau_{k} \notin v
\end{aligned}
$$

Intuitively, this says that we substitute any values stored at a $\langle k \Leftarrow v\rangle$ through for the corresponding $\tau_{k}$. Note that this need not substitute for all the indirect reference identifiers in $C$. It is clear that the above definitions are only partial. For example, if $C$ contains an occurrence of $\langle k \Leftarrow v\rangle$ for which $\tau_{k}$ occurs in $v$, then $\langle\langle C\rangle\rangle$ is undefined. In order to identify for which terms the merge is defined we make use of the notion of reference graph: For a term $C$ we define the graph $\operatorname{rg}(C)$ to be the graph which has nodes as the indirect reference identifiers $k$ in $C$ and edges

$$
k \mapsto l \quad \text { if } \tau_{l} \in v \text { for }\langle k \Leftarrow v\rangle \text { in } C
$$

Proposition 3.3. $\langle\langle\cdot\rangle\rangle$ is a well-defined partial function such that $\langle\langle C\rangle\rangle$ is defined if and only if $\operatorname{rg}(C)$ is acyclic.

Proof. Given in Appendix B] 


$$
\begin{gathered}
\frac{\Delta \vdash v: T \text { a base type }}{(\Delta ; \Theta \vdash a(x: T) P) \stackrel{a\langle v\rangle ?}{\longrightarrow}(\Delta ; \Theta \vdash(x: T) P \cdot v)} \\
\frac{\Theta(k)=T \quad \Delta \vdash w: T \text { a base type }}{(\Delta ; \Theta \vdash\langle k \Leftarrow v\rangle) \stackrel{k\langle w\rangle ?}{\longrightarrow}(\Delta ; \Theta \vdash v \cdot w \|\langle k \Leftarrow v\rangle)} \\
\frac{\Delta \vdash v: T \text { a base type }}{(\Delta ; \Theta \vdash a\langle v\rangle P) \stackrel{a\langle v\rangle !}{\longrightarrow}(\Delta ; \Theta \vdash P)} \\
\frac{\Theta(k)=T \quad T \text { a base type }}{\left(\Delta ; \Theta \vdash \tau_{k} \cdot v\right) \stackrel{k\langle v\rangle !}{\longrightarrow}(\Delta ; \Theta \vdash \mathbf{0})}
\end{gathered}
$$

Figure 5: Basic first-order labelled transition rules

Lemma 3.4 (Composition/Decomposition). For $\Delta ; \Theta \vdash C, D$

(i) If $\langle\langle C \| D\rangle\rangle \equiv E$ and

$$
(\Delta ; \Theta \vdash C) \stackrel{\alpha}{\longrightarrow}\left(\Delta, \Delta^{\prime} ; \Theta, \Theta^{\prime} \vdash C^{\prime}\right) \quad \text { and } \quad(\Delta ; \Theta \vdash D) \stackrel{\bar{\alpha}}{\longrightarrow}\left(\Delta, \Delta^{\prime} ; \Theta, \Theta^{\prime} \vdash D^{\prime}\right)
$$

then there exists a $E^{\prime}$ such that $E \Longrightarrow E^{\prime}$ and $\left\langle\left\langle v^{\prime} .\left(C^{\prime} \| D^{\prime}\right)\right\rangle\right\rangle=E^{\prime}$

(ii) If $\langle\langle C\rangle\rangle \equiv E$ and $C \rightarrow C^{\prime}$ then there exists a $E^{\prime}$ such that $E \rightarrow E^{\prime}$ and $\left\langle\left\langle C^{\prime}\right\rangle\right\rangle \equiv E^{\prime}$

(iii) If $\langle\langle C \| D\rangle\rangle \equiv E$ and $E \rightarrow E^{\prime}$ then one of the following hold

$$
\begin{aligned}
& C \longrightarrow C^{\prime} \text { with }\left\langle\left\langle C^{\prime} \| D\right\rangle\right\rangle \equiv E^{\prime} \\
& \text { or } D \longrightarrow D^{\prime} \text { with }\left\langle\left\langle C \| D^{\prime}\right\rangle\right\rangle \equiv E^{\prime} \\
& \text { or }(\Delta ; \Theta \vdash C) \stackrel{\alpha}{=}\left(\Delta, \Delta^{\prime} ; \Theta, \Theta^{\prime} \vdash C^{\prime}\right) \text { and }(\Delta ; \Theta \vdash D) \stackrel{\bar{\alpha}}{\longrightarrow}\left(\Delta, \Delta^{\prime} ; \Theta, \Theta^{\prime} \vdash D^{\prime}\right) \text { with } \\
& \quad\left\langle\left\langle v \Delta^{\prime} .\left(C^{\prime} \| D^{\prime}\right)\right\rangle\right\rangle \equiv E^{\prime} .
\end{aligned}
$$

Proof. Part (ii) is straightforward as the merge operator $\langle\langle\rangle\rangle$ simply removes subterm of the form $\langle k \Leftarrow v\rangle$, which can't be involved in reductions, and substitutes higher-order values through for variables of higher-order type. Reductions are based on structure alone except for the conditionals which can be affected by first-order substitutions of channel names only.

To show (i) we must consider all the possible cases for $\alpha$. By symmetry there are four distinct pairs of complementary actions. We only consider the cases where $\alpha$ is $v k . a\left\langle\tau_{k}\right\rangle$ ? and $v l . k\left\langle\tau_{l}\right\rangle$ ? as the first-order actions can be treated similarly.

Case: $\Delta ; \Theta \vdash C \stackrel{v k . a\left\langle\tau_{k}\right\rangle ?}{\longrightarrow} \Delta ; \Theta, k: U \vdash C^{\prime}$ and $\Delta ; \Theta \vdash D \stackrel{v k . a\left\langle\tau_{k}\right\rangle !}{\longrightarrow} \Delta ; \Theta, k: U \vdash D^{\prime}$. By inspection we see that

$$
\begin{aligned}
& -C \equiv v \Delta^{\prime} .\left(a(x: T) P \| C^{\prime \prime}\right) \quad \text { with } T \sim_{i s o} U \rightarrow \diamond \\
& -C^{\prime} \equiv v \Delta^{\prime} .\left((x: T) P \cdot \tau_{k} \| C^{\prime \prime}\right) \\
& -D \equiv v \Delta^{\prime \prime} .\left(a\langle v\rangle Q \| D^{\prime \prime}\right) \\
& -D^{\prime} \equiv v \Delta^{\prime \prime} .\left(\langle k \Leftarrow v\rangle\|Q\| D^{\prime \prime}\right)
\end{aligned}
$$


It is easy to see that $\langle\langle C \| D\rangle\rangle \rightarrow\left\langle\left\langle v \Delta^{\prime}, \Delta^{\prime \prime} .\left((x: T) P \cdot v\left\|C^{\prime \prime}\right\| Q \| D^{\prime \prime}\right)\right\rangle\right\rangle$ let us call the target of this reduction $E^{\prime}$. We simply need to check

$$
\begin{aligned}
E^{\prime} & \equiv\left\langle\left\langle v \Delta^{\prime}, \Delta^{\prime \prime} .\left((x: T) P \cdot v\left\|C^{\prime \prime}\right\| Q \| D^{\prime \prime}\right)\right\rangle\right\rangle \\
\left(\tau_{k} \notin v\right) \quad & \equiv\left\langle\left\langle v \Delta^{\prime} .\left((x: T) P \cdot \tau_{k} \| C^{\prime \prime}\right) \| v \Delta^{\prime \prime} .\left(\langle k \Leftarrow v\rangle\|Q\| D^{\prime \prime}\right)\right\rangle\right\rangle \\
& \equiv\left\langle\left\langle C^{\prime} \| D^{\prime}\right\rangle\right\rangle
\end{aligned}
$$

Case: $\Delta ; \Theta \vdash C \stackrel{v l . k\left\langle\tau_{l}\right\rangle ?}{\longrightarrow} \Delta ; \Theta, l: T \vdash C^{\prime}$ and $\Delta ; \Theta \vdash D \stackrel{v l . k\left\langle\tau_{l}\right\rangle !}{\longrightarrow} \Delta ; \Theta, l: T \vdash D^{\prime}$. Again, by inspection we see that

$$
\begin{aligned}
& -C \equiv v \Delta^{\prime} .\left(\langle k \Leftarrow v\rangle \| C^{\prime \prime}\right) \\
& -C^{\prime} \equiv v \Delta^{\prime} .\left(v \cdot \tau_{l}\|\langle k \Leftarrow v\rangle\| C^{\prime \prime}\right) \\
& -D \equiv v \Delta^{\prime \prime} .\left(\tau_{k} \cdot w \| D^{\prime \prime}\right) \\
& -D^{\prime} \equiv v \Delta^{\prime \prime} .\left(\langle l \Leftarrow w\rangle \| D^{\prime \prime}\right)
\end{aligned}
$$

Note that the previous proposition tells us that $\operatorname{rg}(C \| D)$ must be acyclic - in particular, $\tau_{k} \notin v$. Here we see that

$$
\begin{aligned}
\langle\langle C \| D\rangle\rangle & \equiv\left\langle\left\langle v \Delta^{\prime}, \Delta^{\prime \prime} \cdot\left(\langle k \Leftarrow v\rangle\left\|C^{\prime \prime}\right\| \tau_{k} \cdot w \| D^{\prime \prime}\right)\right\rangle\right\rangle \\
\left(\tau_{k} \notin v\right) & \equiv\left\langle\left\langle v \Delta^{\prime}, \Delta^{\prime \prime} \cdot\left(\langle k \Leftarrow v\rangle\left\|C^{\prime \prime}\right\| v \cdot w \| D^{\prime \prime}\right)\right\rangle\right\rangle \\
\left(\tau_{l} \notin v, w, C^{\prime \prime}, D^{\prime \prime}\right) & \equiv\left\langle\left\langle v \Delta^{\prime}, \Delta^{\prime \prime} \cdot\left(\langle k \Leftarrow v\rangle\left\|C^{\prime \prime}\right\| v \cdot \tau_{l}\|\langle l \Leftarrow w\rangle\| D^{\prime \prime}\right)\right\rangle\right\rangle \\
& \equiv\left\langle\left\langle C^{\prime} \| D^{\prime}\right\rangle\right\rangle
\end{aligned}
$$

So by letting $E^{\prime}$ be $\left\langle\left\langle C^{\prime} \| D^{\prime}\right\rangle\right\rangle$ we note that $\langle\langle C \| D\rangle\rangle \Longrightarrow E^{\prime}$ as required.

To show (iii) we suppose $\langle\langle C \| D\rangle\rangle \equiv E$ and that $E \longrightarrow E^{\prime}$. We must consider all possible ways in which this reduction can occur. If the reduction arises from a conditional then it is clear that we must have $C \rightarrow C^{\prime}$ or $D \rightarrow D^{\prime}$ for some $C^{\prime}$ or $D^{\prime}$. Moreover it is easy to check that $\left\langle\left\langle C^{\prime} \| D\right\rangle\right\rangle$ (resp $\left.\left\langle\left\langle C \| D^{\prime}\right\rangle\right\rangle\right) \equiv E^{\prime}$. There are two more possibilities to consider:

Case: the reduction arises from a $\beta$-reduction. In this case either $C \rightarrow C^{\prime}$ or $D \rightarrow D^{\prime}$ as above and the result follows easily, or $v$ is $(x: U) P$ and

$-C \equiv v \Delta^{\prime} .\left(\tau_{k} \cdot w \| C^{\prime \prime}\right) \quad$ with all names in $\Delta^{\prime}$ appearing in $w$

- $D \equiv v \Delta^{\prime \prime} \cdot\left(\langle k \Leftarrow v\rangle \| D^{\prime \prime}\right) \quad$ with $\quad \tau_{k} \notin v$

- $E^{\prime} \equiv\left\langle\left\langle v \Delta^{\prime}, \Delta^{\prime \prime} .\left(P[w / x]\left\|C^{\prime \prime}\right\|\langle k \Leftarrow v\rangle \| D^{\prime \prime}\right)\right\rangle\right\rangle$

or a symmetric version of these with the roles of $C$ and $D$ reversed. So we notice that if $U \sim_{\text {iso }} T \rightarrow \diamond$, we have

$$
\Delta ; \Theta \vdash C \stackrel{v l . k\left\langle\tau_{l}\right\rangle !}{\longrightarrow} \Delta ; \Theta, l: T \vdash C^{\prime} \quad \text { and } \quad \Delta ; \Theta \vdash D \stackrel{v l . k\left\langle\tau_{l}\right\rangle ?}{\longrightarrow} \Delta ; \Theta, l: T \vdash D^{\prime}
$$

where $C^{\prime} \equiv v \Delta^{\prime} .\left(\langle l \Leftarrow w\rangle \| C^{\prime \prime}\right)$ and $D^{\prime} \equiv v \Delta^{\prime \prime} .\left(P\left[\tau_{l} / x\right]\|\langle k \Leftarrow v\rangle\| D^{\prime \prime}\right)$. We check:

$$
\begin{aligned}
\left\langle\left\langle C^{\prime} \| D^{\prime}\right\rangle\right\rangle & \equiv\left\langle\left\langle v \Delta^{\prime} .\left(\langle l \Leftarrow w\rangle \| C^{\prime \prime}\right)\left\|v \Delta^{\prime \prime} .\left(P\left[\tau_{k} / x\right]\right)\right\|\langle k \Leftarrow v\rangle \| D^{\prime \prime}\right\rangle\right\rangle \\
\left(\tau_{l} \notin v, w, C^{\prime \prime}, D^{\prime \prime}\right) & \equiv\left\langle\left\langle v \Delta^{\prime}, \Delta^{\prime \prime} .\left(C^{\prime \prime}\|P[w / x]\|\langle k \Leftarrow v\rangle \| D^{\prime \prime}\right)\right\rangle\right\rangle \\
& \equiv E^{\prime}
\end{aligned}
$$

as required. Alternatively, it could be that $U$ is a base type, in which case

$$
\Delta ; \Theta \vdash C \stackrel{v \Delta^{\prime} \cdot k\langle w\rangle !}{\longrightarrow} \Delta, \Delta^{\prime} ; \Theta \vdash C^{\prime} \quad \text { and } \quad \Delta ; \Theta \vdash D \stackrel{v \Delta^{\prime} \cdot k\langle w\rangle ?}{\Longrightarrow} \Delta, \Delta^{\prime} ; \Theta \vdash D^{\prime}
$$

where $C^{\prime} \equiv C^{\prime \prime}$ and $D^{\prime} \equiv v \Delta^{\prime \prime} .\left(P[w / x]\|\langle k \Leftarrow v\rangle\| D^{\prime \prime}\right)$. It is easy to check that $\left\langle\left\langle C^{\prime} \| D^{\prime}\right\rangle\right\rangle \equiv E^{\prime}$ as required. 
Case: the reduction arises from communication. Again we see that either $C \longrightarrow C^{\prime}$ or $D \longrightarrow D^{\prime}$, in which case we easily obtain the result, or

$-C \equiv v \Delta^{\prime} .\left(a\langle v\rangle P \| C^{\prime \prime}\right)$

$-D \equiv v \Delta^{\prime \prime} .\left(a(x: T) Q \| D^{\prime \prime}\right)$

- $E^{\prime} \equiv\left\langle\left\langle v \Delta^{\prime} .\left(P \| C^{\prime \prime}\right) \| v \Delta^{\prime \prime} .\left((x: T) Q \cdot v \| D^{\prime \prime}\right)\right\rangle\right\rangle$

or a symmetric version of this with the roles of $C$ and $D$ reversed. Again we must consider whether the type $T$ is a base type or higher-order. We omit the details of the former case. Suppose then that $\Delta ; \Theta \vdash v: T \sim_{i s o} U \rightarrow \diamond$ we know

$$
\begin{aligned}
& \Delta ; \Theta \vdash C \stackrel{v k . a\left\langle\tau_{k}\right\rangle !}{\longrightarrow} \Delta ; \Theta, k: U \vdash C^{\prime} \quad \text { and } \quad \Delta ; \Theta \vdash D \stackrel{v k . a\left\langle\tau_{k}\right\rangle ?}{\longrightarrow} \Delta ; \Theta, k: U \vdash D^{\prime} \\
& \text { where } C^{\prime} \equiv v \Delta^{\prime} .\left(\langle k \Leftarrow v\rangle\|P\| C^{\prime \prime}\right) \text { and } D^{\prime} \equiv v \Delta^{\prime \prime} .\left((x: T) Q \cdot \tau_{k} \| D^{\prime \prime}\right) \text {. We check: } \\
& \begin{aligned}
\left\langle\left\langle C^{\prime} \| D^{\prime}\right\rangle\right\rangle & \equiv\left\langle\left\langle v \Delta^{\prime} .\left(\langle k \Leftarrow v\rangle\|P\| C^{\prime \prime}\right) \| v \Delta^{\prime \prime} .\left((x: T) Q \cdot \tau_{k} \| D^{\prime \prime}\right)\right\rangle\right\rangle \\
\left(\tau_{k} \notin v, P, C^{\prime \prime}, D^{\prime \prime}\right) \quad & \equiv\left\langle\left\langle v \Delta^{\prime}, \Delta^{\prime \prime} .\left(P\left\|C^{\prime \prime}\right\|(x: T) Q \cdot v \| D^{\prime \prime}\right)\right\rangle\right\rangle \\
& \equiv E^{\prime}
\end{aligned}
\end{aligned}
$$

as required.

Definition 3.5. Let $\approx_{m}$ be defined to be

$\Delta ; \Theta \models\left\langle\left\langle C_{1} \| D\right\rangle\right\rangle \approx_{m}\left\langle\left\langle C_{2} \| D\right\rangle\right\rangle \quad$ if and only if $\quad \Delta ; \Theta \models C_{1} \approx C_{2} \quad$ and $\quad \Delta ; \Theta \vdash D$ whenever $\left\langle\left\langle C_{1} \| D\right\rangle\right\rangle$ and $\left\langle\left\langle C_{2} \| D\right\rangle\right\rangle$ are defined.

Note that in the case where $\Theta$ is empty we have that $\left\langle\left\langle C_{i} \| D\right\rangle\right\rangle=C_{i} \| D$, and hence $\approx_{m}$ and $\cong_{p}$ coincide.

Lemma 3.6. $\approx_{m}$ is reduction-closed.

Proof. Follows easily from the previous lemma. Take $\Delta ; \Theta \models\left\langle\left\langle C_{1} \| D\right\rangle\right\rangle \approx_{m}\left\langle\left\langle C_{2} \| D\right\rangle\right\rangle$ and suppose $\left\langle\left\langle C_{1} \| D\right\rangle\right\rangle \rightarrow E$. We must show that $\left\langle\left\langle C_{2} \| D\right\rangle\right\rangle \rightarrow E^{\prime}$ for some $E^{\prime}$ such that $\Delta ; \Theta \models E \approx_{m} E^{\prime}$. We know from Part (iii) of the previous lemma that one of three cases must hold. Either, $C_{1} \longrightarrow C_{1}^{\prime}$, $D \longrightarrow D^{\prime}$ or there are complementary actions from both $C_{1}$ and $D$. We only deal with the last case as the others follow easily from the hypothesis that $\Delta ; \Theta \models C_{1} \approx C_{2}$ and Part (ii) of the previous lemma.

We have then that $\Delta ; \Theta \vdash C_{1} \stackrel{\alpha}{=} \Rightarrow \Delta, \Delta^{\prime} ; \Theta, \Theta^{\prime} \vdash C_{1}^{\prime}$ and $\Delta ; \Theta \vdash D \stackrel{\bar{\alpha}}{=} \Rightarrow \Delta, \Delta^{\prime} ; \Theta, \Theta^{\prime} \vdash D^{\prime}$ such that $E \equiv\left\langle\left\langle C_{1}^{\prime} \| D^{\prime}\right\rangle\right\rangle$. We know by hypothesis that there must exist some

$$
\Delta ; \Theta \vdash C_{2} \stackrel{\alpha}{\Longrightarrow} \Delta, \Delta^{\prime} ; \Theta, \Theta^{\prime} \vdash C_{2}^{\prime}
$$

such that

$$
\Delta, \Delta^{\prime} ; \Theta, \Theta^{\prime} \models C_{1}^{\prime} \approx C_{2}^{\prime} .
$$

We can now use Parts (i) and (ii) of the previous lemma to see that $\left\langle\left\langle C_{2} \| D\right\rangle\right\rangle \Longrightarrow E^{\prime}$ such that $E^{\prime} \equiv\left\langle\left\langle C_{2}^{\prime} \| D^{\prime}\right\rangle\right\rangle$. Note that $(\dagger)$ guarantees $\Delta ; \Theta \models E \approx_{m} E^{\prime}$ to finish. 
Theorem 3.7. For all closed terms $P, Q$ of $\mathrm{HO} \pi$ :

$$
\Delta \models P \approx Q \text { implies } \Delta \models P \cong_{p} Q
$$

Proof. We let $\approx_{p}$ denote the relation

$$
\Delta, \Delta^{\prime} \models(P \| R) \approx_{p}(Q \| R) \text { iff } \Delta \models P \approx Q \text { and } \Delta, \Delta^{\prime} \vdash R
$$

It is easy to see that $\approx_{p}$ is a $\|$-contextual relation over terms of HO $\pi$. It is also easy to see that $\approx_{p}$ is symmetric and barb preserving and coincides with $\approx_{m}$ for closed terms of $\mathrm{HO} \pi$, thus Lemma 3.6 can be instantiated to demonstrate that $\approx_{p}$ is reduction-closed and, given that $\cong_{p}$ is defined to be the largest symmetric, $\|$-contextual, reduction-closed, and barb-preserving relation over terms of $\mathrm{HO} \pi$, then we have our result.

Corollary 3.8 (Soundness). For all terms $P, Q$ of $H O \pi$ :

$$
\Gamma \models P \approx^{o} Q \text { implies } \Gamma \models P \cong Q
$$

Proof. Follows from the previous theorem and Lemma 2.2

3.4. Completeness of bisimilarity for contextual equivalence. The interactions described by the labelled transition system are not obviously derived by genuine contextual observations in HO $\pi$ because of the use of the extra syntax for indirect references. In order to show completeness of our bisimilarity for contextual equivalence we must demonstrate that the indirect references are in fact definable as terms of the language proper. Following Sangiorgi [13], we implement the implicit protocol outlined by the indirect references by using the following translation of the augmented terms into $\mathrm{HO} \pi$ :

$$
\begin{aligned}
{\left[\left[k_{1}: T_{1}, \ldots, k_{n}: T_{n}\right]\right] } & =k_{1}: \operatorname{ch}\left[T_{1}\right], \ldots, k_{n}: \operatorname{ch}\left[T_{n}\right] & \\
{[[\Gamma ; \Theta \vdash C]] } & =\Gamma,[[\Theta]] \vdash\left[[C]_{\Theta}\right. & \\
{\left[\left[\tau_{k}\right]\right]_{\Theta} } & =(x: T) k\langle x\rangle \mathbf{0} & \text { if } \Theta(k)=T \\
{[\langle k \Leftarrow v\rangle]_{\Theta} } & =* k[[v]]_{\Theta} &
\end{aligned}
$$

The translation acts homomorphically on all other terms. We abuse notation here by using identifiers $k$ as channel names in the translation. It is evident that this translation is well-defined in the sense that the translation of well-typed augmented terms are indeed well-typed terms of $\mathrm{HO} \pi$.

We would now like to prove a correspondence between reductions from the terms of the augmented syntax and reductions between their translations. However, we note that in translating a term containing both $\langle k \Leftarrow v\rangle$ and $\tau_{k}$ we provide matching input and output prefixes, which, in $\mathrm{HO} \pi$ may create a communication which was not possible in the source term. This turns out not to be of particular concern to us though as we see that if we starting with terms of $\mathrm{HO} \pi$, then terms reachable by transitions are balanced in the following sense: we call a term $C$ of the augmented language balanced if for each $k$ then $C$ contains at most one of $\tau_{k}$ (possible multiple times) or $\langle k \Leftarrow v\rangle$. Unfortunately the translation may introduce extra reductions which aren't present in the source term. These arise through the translation of terms of the form $\tau_{k} \cdot v$. Note that

$$
\left[\left[\tau_{k} \cdot v\right]\right]=(x: T) k\langle x\rangle \mathbf{0} \cdot[[v]] \stackrel{\tau}{\longrightarrow} k\langle[[v]\rangle\rangle \mathbf{0}
$$

but $\tau_{k} \cdot v$ has no corresponding reduction. We will identify these rogue reductions as housekeeping reductions and indicate them with $\stackrel{\mathrm{h}}{\longrightarrow}$ defined as any reduction which can be derived using the axiom

$$
(\mathrm{h}-\mathrm{redn}) \quad(x: T) k\langle x\rangle \mathbf{0} \cdot v \rightarrow k\langle v\rangle \mathbf{0}
$$

Lemma 3.9. If $\Delta ; \Theta \vdash C$ is balanced then 
(1) If $C \Longrightarrow C^{\prime}$ then $\left[[C]_{\Theta} \Longrightarrow\left[\left[C^{\prime}\right]_{\Theta}\right.\right.$

(2) If $\left[[C]_{\Theta} \Longrightarrow P\right.$ then $\left[[C]_{\Theta} \Longrightarrow[[D]]_{\Theta} \stackrel{\mathrm{h}}{\rightarrow} * P\right.$ for some $\Delta$; $\Theta \vdash D$ such that $C \Longrightarrow D$.

Proof. We will omit mention of the environment $\Theta$ in the proof as it plays no role. Part 1 is straightforward. For Part 2 we use induction on the length of the reductions. If there are no reductions then we are done. We examine the base case in which $[[C]] \rightarrow P$. If this reduction happens to be a housekeeping move, that is, $[[C] \stackrel{\mathrm{h}}{\longrightarrow} P$ then there is nothing to prove. Suppose otherwise, then it is not too difficult to check that $P \equiv[[D]$ for some $D$ such that $C \rightarrow D$. For the inductive case suppose that

$$
[[C]] \longrightarrow P
$$

By inspecting the translation $[[\cdot]]$ and using the fact that $C$ is balanced we see that

$$
[[C] \stackrel{\mathrm{h}}{\rightarrow} \rightarrow Q \quad \text { implies } \quad[[C]] \rightarrow \stackrel{\mathrm{h}}{\rightarrow} Q
$$

thus we may assume that the first reduction in $(\dagger)$ above is not of the form $\stackrel{h}{\longrightarrow}$. This means that $[[C]] \longrightarrow\left[\left[C^{\prime}\right]\right] P$ for some $C^{\prime}$ such that $C \longrightarrow C^{\prime}$. It is clear that $C^{\prime}$ is also balanced so we may apply the inductive hypothesis to

$$
\left[\left[C^{\prime}\right]\right] \Longrightarrow P
$$

to obtain a $D$ such that $C^{\prime} \Longrightarrow D^{\prime}$ and $\left[\left[C^{\prime}\right]\right] \Longrightarrow[[D]] \stackrel{\mathrm{h}}{\rightarrow}^{*} P$. Putting these together we obtain

$$
C \rightarrow C^{\prime} \Longrightarrow D \quad \text { and } \quad[[C]] \longrightarrow\left[\left[C^{\prime}\right]\right] \Longrightarrow\left[[D] \stackrel{\mathrm{h}}{\rightarrow}^{*} P\right.
$$

as required.

When $\Delta^{\prime}$ is of length at most one, we shall write $\delta\left\langle\Delta^{\prime}\right\rangle$ as shorthand, defined:

$$
\delta\langle\emptyset\rangle=\delta\langle\cdot\rangle \quad \delta\langle a: T\rangle=\delta\langle a\rangle
$$

Moreover, note that whenever $(\Delta ; \Theta \vdash D) \stackrel{\alpha}{\Longrightarrow}\left(\Delta, \Delta^{\prime} ; \Theta, \Theta^{\prime} \vdash D^{\prime}\right)$, we have that $\Delta^{\prime}$ has at length most one, and so $\delta\left\langle\Delta^{\prime}\right\rangle$ is well-defined.

Proposition 3.10. For each $\alpha, \Delta$ and fresh channels $\delta, \delta^{\prime}$ of appropriate type given by $\alpha$ and $\Delta$, there exists a process $\mathcal{T}_{\alpha}^{\Delta}$ (defined in Figure 6 in $\mathrm{HO} \pi$ such that if

$$
\Delta ; \Theta \vdash C \stackrel{\alpha}{\longrightarrow} \Delta, \Delta^{\prime} ; \Theta, \Theta^{\prime} \vdash C^{\prime}
$$

then

$$
\Delta,\left[\left[\Theta, \Theta^{\prime}\right]\right], \delta: \operatorname{ch}\left[T_{0}\right], \delta^{\prime}: \operatorname{ch}[\cdot] \vdash \mathcal{T}_{\alpha}^{\Delta,[\Theta \Theta]}
$$

and moreover, for balanced $D$

$$
(\Delta ; \Theta \vdash D) \stackrel{\alpha}{\Longrightarrow}\left(\Delta, \Delta^{\prime} ; \Theta, \Theta^{\prime} \vdash D^{\prime}\right)
$$

if and only if $\Delta ; \Theta \vdash D$ and

$$
\mathcal{T}_{\alpha}^{\Delta,[\Theta \rrbracket} \|[[D]]_{\Theta} \Longrightarrow v \Delta^{\prime} .\left(\delta\left\langle\Delta^{\prime}\right\rangle \| P\right) \quad \text { with } \quad\left[\left[D^{\prime}\right]\right]_{\Theta, \Theta^{\prime}} \stackrel{\mathrm{h}}{\rightarrow}{ }^{*} P .
$$


Proof. It is straightforward to check that $\Delta,\left[\left[\Theta, \Theta^{\prime}\right]\right], \delta: \operatorname{ch}\left[T_{0}\right], \delta^{\prime}: \operatorname{ch}[\cdot] \vdash \mathcal{T}_{\alpha}^{\Delta}$ whenever

$$
\Delta ; \Theta \vdash C \stackrel{\alpha}{\longrightarrow} \Delta, \Delta^{\prime} ; \Theta, \Theta^{\prime} \vdash C^{\prime} .
$$

For the remainder, to show the 'only if' direction we use Lemma 3.9 Part 1 to reduce our obligation to the case of a single transition $\stackrel{\alpha}{\longrightarrow}$, and we must consider each label $\alpha$. By way of example we show the case for $\alpha=v l . k\left\langle\tau_{l}\right\rangle$ ! (the other cases can be treated similarly). Suppose:

$$
(\Delta ; \Theta \vdash D) \stackrel{\alpha}{\longrightarrow}\left(\Delta ; \Theta, l: U \vdash D^{\prime}\right) .
$$

then we know that

and

$$
D \equiv v \Delta^{\prime \prime} \cdot\left(\tau_{k} \cdot v \| D^{\prime \prime}\right)
$$

(1)

$$
D^{\prime} \equiv v \Delta^{\prime \prime} .\left(\langle l \Leftarrow v\rangle \| D^{\prime \prime}\right) .
$$

We see that for $T \sim_{i s o} U \rightarrow \diamond$

$$
\begin{aligned}
\mathcal{T}_{\alpha}^{\Delta,[\Theta \rrbracket} \|\left[[D]_{\Theta}\right. & \equiv k(x: T)\left(* l(y: U) x \cdot y \|\left(\boldsymbol{\delta}\langle\rangle \oplus \boldsymbol{\delta}^{\prime}\langle\rangle\right)\right) \| v \Delta^{\prime \prime} \cdot\left(((z: T) k\langle z\rangle \mathbf{0}) \cdot[v v]_{\Theta} \|\left[\left[D^{\prime \prime}\right]_{\Theta}\right)\right. \\
& \Longrightarrow\left(\delta\langle\rangle \oplus \boldsymbol{\delta}^{\prime}\langle\rangle\right) \| v \Delta^{\prime \prime} .\left(* l(y: U)[[v]]_{\Theta} \cdot y \|\left[\left[D^{\prime \prime}\right]_{\Theta}\right)\right. \\
& \Longrightarrow \delta\langle\rangle \|\left[D^{\prime}\right]_{\Theta, l: U}
\end{aligned}
$$

as required.

For the converse direction we suppose that

$$
\mathcal{T}_{\alpha}^{\Delta,[\Theta \rrbracket} \|\left[[D]_{\Theta} \Longrightarrow v^{\prime} .\left(\delta\left\langle\Delta^{\prime}\right\rangle \| P\right)\right.
$$

Again, we must perform a case analysis on $\alpha$. We show the case in which $\alpha$ is $v l . k\left\langle\tau_{l}\right\rangle$ ? (the other cases can be treated similarly). We know $\Delta^{\prime}$ is empty so $\mathcal{T}_{\alpha}^{\Delta, \llbracket \Theta \rrbracket}\left\|[[D]]_{\Theta} \Longrightarrow \delta\langle\rangle\right\| P$. Note that $\mathcal{T}_{\alpha}^{\Delta,[\Theta]}$ has no reductions of its own and can only interact with $[[D]]_{\Theta}$ so we can detail the assumed reductions as

$$
\mathcal{T}_{\alpha}^{\Delta,[\Theta \rrbracket}\left\|[[D]]_{\Theta} \Longrightarrow \mathcal{T}_{\alpha}^{\Delta, \llbracket \Theta \rrbracket}\right\| P_{0} \longrightarrow\left(\delta\langle\rangle \oplus \boldsymbol{\delta}^{\prime}\langle\rangle\right)\left\|P_{1} \Longrightarrow \delta\langle\rangle\right\| P
$$

where $[[D]] \Longrightarrow P_{0}$ and $P_{1} \Longrightarrow P$. We assumed that $D$ is balanced so Lemma3.9 Part 2 applied to $[[D]] \Longrightarrow P_{0}$ tells us that $[[D]] \Longrightarrow\left[D_{0}\right]_{\Theta} \stackrel{\mathrm{h}}{\longrightarrow}{ }^{*} P_{0}$ for some $D_{0}$ such that $D \Longrightarrow D_{0}$. We know that $P_{0}$ is obtained from $\left[\left[D_{0}\right]_{\Theta}\right.$ by housekeeping reductions and that it interacts with $\mathcal{T}_{\alpha}^{\Delta}$. This tells us that we must have the forms

and

$$
P_{0} \equiv v \Delta^{\prime \prime} .\left(* k[[v]]_{\Theta} \| P_{0}^{\prime}\right)
$$

This in turn tells us that

$$
P_{1} \equiv v \Delta^{\prime \prime} \cdot\left([[v]]_{\Theta} \cdot\left[\left[\tau_{l}\right]\right]_{\Theta, l: U}\left\|* k[[v]]_{\Theta}\right\| P_{0}^{\prime}\right)
$$

$$
D_{0} \equiv v \Delta^{\prime \prime} .\left(\langle k \Leftarrow v\rangle \| D_{0}^{\prime}\right)
$$

such that $\left[\left[D_{0}^{\prime}\right]\right]_{\Theta} \stackrel{\mathrm{h}}{\rightarrow}{ }^{*} P_{0}^{\prime}$. Now it is clear that

$$
\left.\left(\Delta ; \Theta \vdash D_{0}\right) \stackrel{v l . k\left\langle\tau_{l}\right\rangle ?}{\longrightarrow}(\Delta ; \Theta, l: U) \vdash D_{1}\right)
$$




$$
\begin{array}{llll}
\mathcal{T}_{d\langle v\rangle ?}^{\Delta} & =d\langle v\rangle\left(\boldsymbol{\delta}\langle\rangle \oplus \boldsymbol{\delta}^{\prime}\langle\rangle\right) & & \\
\mathcal{T}_{d\langle v\rangle !}^{\Delta} & =d(x: T) \text { if } x=v \text { then }\left(\boldsymbol{\delta}\langle\rangle \oplus \boldsymbol{\delta}^{\prime}\langle\rangle\right) \text { else } \mathbf{0} & & \text { where } \Delta(d)=\operatorname{ch}[T] \\
\mathcal{T}_{v b . d}^{\Delta} d\langle b\rangle ? & =v b: T .\left(d\langle b\rangle\left(\boldsymbol{\delta}\langle b\rangle \oplus \boldsymbol{\delta}^{\prime}\langle\rangle\right)\right) & & \text { where } \Delta(d)=\operatorname{ch}[T] \\
\mathcal{T}_{v b . d}^{\Delta} d\langle b\rangle ! & =d(x: T) \text { if } x \notin \Delta \text { then }\left(\boldsymbol{\delta}\langle x\rangle \oplus \boldsymbol{\delta}^{\prime}\langle\rangle\right) \text { else } \mathbf{0} & & \text { where } \Delta(d)=\operatorname{ch}[T] \\
\mathcal{T}_{v k . d}^{\Delta} d\left\langle\tau_{k}\right\rangle ? & =d\langle(x: U) k\langle x\rangle \mathbf{0}\rangle\left(\boldsymbol{\delta}\langle\rangle \oplus \boldsymbol{\delta}^{\prime}\langle\rangle\right) & & \text { where } \Delta(d)=\operatorname{ch}[T] \text { and } T \sim_{i s o} U \rightarrow \diamond \\
\mathcal{T}_{v k . d\left\langle\tau_{k}\right\rangle !}^{\Delta}=d(x: T)\left(* l(y: U) x \cdot y \|\left(\boldsymbol{\delta}\langle\rangle \oplus \boldsymbol{\delta}^{\prime}\langle\rangle\right)\right) & & \text { where } \Delta(d)=\operatorname{ch}[T] \text { and } T \sim_{\text {iso }} U \rightarrow \diamond
\end{array}
$$

$\oplus$ represents an encoding of internal choice in $\mathrm{HO} \pi$

$$
\text { if } x \notin \emptyset \text { then } P \text { else } Q=P
$$

if $x \notin(a: T, \Delta)$ then $P$ else $Q=$ if $x=a$ then $Q$ else if $x \notin \Delta$ then $P$ else $Q$

Figure 6: Testing processes for labelled transitions

where $D_{1} \equiv v \Delta^{\prime \prime} .\left(v \cdot \tau_{l}\|\langle k \Leftarrow v\rangle\| D_{0}^{\prime}\right)$. We check

$$
\begin{array}{rll}
{\left[\left[D_{1}\right]\right]_{\Theta, l: U}} & \equiv v \Delta^{\prime \prime} \cdot\left([[v]]_{\Theta} \cdot\left[\left[\tau_{l}\right]\right]_{\Theta, l: U}\|* k[[v]]\|\left[\left[D_{0}^{\prime}\right]\right]_{\Theta}\right) \\
& \stackrel{\mathrm{h}^{*}}{\longrightarrow} & v \Delta^{\prime \prime} .\left(\left[[v]_{\Theta} \cdot\left[\left[\tau_{l}\right]_{\Theta, l: U}\|* k[[v]]\| P_{0}^{\prime}\right)\right.\right. \\
& \equiv & P_{1} \\
& \longrightarrow & P
\end{array}
$$

Therefore $\left[\left[D_{1}\right]\right]=\Rightarrow P$ and we can apply Lemma 3.9 Part 2 to this to see that $\left[\left[D_{1}\right]\right]=\Rightarrow\left[\left[D^{\prime}\right]\right] \stackrel{\mathrm{h}}{\rightarrow}{ }^{*} P$ for some $D^{\prime}$ such that $D_{1} \Longrightarrow D^{\prime}$. By collecting the above together we obtain

$$
(\Delta ; \Theta \vdash D) \Longrightarrow\left(\Delta ; \Theta \vdash D_{0}\right) \stackrel{\alpha}{\longrightarrow}\left(\Delta ; \Theta, l: U \vdash D_{1}\right) \Longrightarrow\left(\Delta ; \Theta, l: U \vdash D^{\prime}\right)
$$

with $\left[\left[D^{\prime}\right]\right]_{\Theta, l: U} \stackrel{\mathrm{h}}{\rightarrow} P$ as required.

Lemma 3.11 (Extrusion). If $\Delta \models v \Delta^{\prime} .\left(\delta\left\langle\Delta^{\prime}\right\rangle \| P\right) \cong{ }_{p} v \Delta^{\prime} .\left(\delta\left\langle\Delta^{\prime}\right\rangle \| Q\right)$ then $\Delta, \Delta^{\prime} \models P \cong{ }_{p} Q$.

Proof. Follows a similar argument found in [7]: define a relation $\mathcal{R}$ such that

$$
\Delta, \Delta^{\prime} \models P R Q \quad \text { iff } \quad \Delta \models v \Delta^{\prime} .\left(\delta\left\langle\Delta^{\prime}\right\rangle \| P\right) \cong_{p} v \Delta^{\prime} .\left(\delta\left\langle\Delta^{\prime}\right\rangle \| Q\right)
$$

and show that $\mathcal{R}$ is barb-preserving, reduction-closed and $\|$-contextual. These properties follow from the corresponding property for $\cong_{p}$ and an extra piece of context to interact with $\delta\left\langle\Delta^{\prime}\right\rangle$.

Theorem 3.12 (Completeness). For all closed terms $P, Q$ of $\mathrm{HO} \pi$ :

$$
\Delta \models P \cong{ }_{p} Q \quad \text { implies } \quad \Delta \models P \approx Q
$$

Proof. We define $\mathcal{R}$ over terms of the augmented language to be

$$
\Delta ; \Theta \models C R D \quad \text { iff } \quad \Delta,[[\Theta]] \models[[C]]_{\Theta} \cong_{p}[[D]]_{\Theta}
$$

and show that $\mathcal{R}$ is a bisimulation. Take $\Delta ; \Theta \models C R D$ and suppose that

$$
(\Delta ; \Theta \vdash C) \stackrel{\alpha}{\longrightarrow}\left(\Delta, \Delta^{\prime} ; \Theta, \Theta^{\prime} \vdash C^{\prime}\right) .
$$

We know from Proposition 3.10 that

$$
\Delta,\left[\left[\Theta, \Theta^{\prime}\right]\right], \delta: \operatorname{ch}\left[T_{0}\right], \delta^{\prime}: \operatorname{ch}[\cdot] \vdash \mathcal{T}_{\alpha}^{\Delta, \llbracket \Theta \rrbracket}
$$

and that

$$
\mathcal{T}_{\alpha}^{\Delta,[\Theta \rrbracket} \|[[C]]_{\Theta} \Longrightarrow v \Delta^{\prime} .\left(\delta\left\langle\Delta^{\prime}\right\rangle \| P\right)
$$


with $\left[\left[C^{\prime}\right]\right]_{\Theta, \Theta^{\prime}} \stackrel{\mathrm{h}}{\rightarrow}$ * $P$. We know that

$$
\Delta,[[\Theta]] \models\left[[C]_{\Theta} \cong{ }_{p}[[D]]_{\Theta}\right.
$$

by the definition of $\mathcal{R}$, and hence, by contextuality we also have

$$
\Delta,\left[\left[\Theta, \Theta^{\prime}\right]\right], \delta: \operatorname{ch}\left[T_{0}\right], \delta^{\prime}: \operatorname{ch}[\cdot] \models \mathcal{T}_{\alpha}^{\Delta,[\Theta \rrbracket} \|\left[[C]_{\Theta} \cong{ }_{p} \mathcal{T}_{\alpha}^{\Delta,[\Theta \rrbracket} \|[[D]]_{\Theta}\right.
$$

This tells us that

such that

$$
\mathcal{T}_{\alpha}^{\Delta,[\Theta]]} \|[[D]]_{\Theta} \Longrightarrow Q^{\prime}
$$

$$
\Delta,\left[\left[\Theta, \Theta^{\prime}\right]\right] \models v \Delta^{\prime} .\left(\delta\left\langle\Delta^{\prime}\right\rangle \| P\right) \cong{ }_{p} Q^{\prime} .
$$

But by the construction of $\mathcal{T}_{\alpha}^{\Delta,[\Theta \rrbracket]}$ we notice that $v \Delta^{\prime} .\left(\delta\left\langle\Delta^{\prime}\right\rangle \| P\right)$ barbs on $\delta$ but not on $\delta^{\prime}$. Therefore, by the preservation of barbs property of $\cong_{p}$, we know that $Q^{\prime}$ must also barb on $\delta$ but not on $\delta^{\prime}$. This constrains $Q^{\prime}$ so that $Q^{\prime} \equiv v \Delta^{\prime} .\left(\delta\left\langle\Delta^{\prime}\right\rangle \| Q\right)$. We apply Lemma 3.9 Part 2 to $\mathcal{T}_{\alpha}^{\Delta,[\Theta \rrbracket} \|[D]_{\Theta} \Longrightarrow Q^{\prime}$ to see that there is some $D^{\prime \prime}$ such that $\mathcal{T}_{\alpha}^{\Delta,[\Theta]]} \|\left[[D]_{\Theta}=\Rightarrow\left[\left[D^{\prime \prime}\right]\right]_{\Theta, \Theta^{\prime}} \stackrel{\mathrm{h}}{\rightarrow}{ }^{*} v \Delta^{\prime} .\left(\delta\left\langle\Delta^{\prime}\right\rangle \| Q\right)\right.$ from which it clearly follows that $D^{\prime \prime} \equiv v \Delta^{\prime} .\left(\delta\left\langle\Delta^{\prime}\right\rangle \| D^{\prime}\right)$ and $\left[\left[D^{\prime}\right]\right]_{\Theta, \Theta^{\prime}} \stackrel{h^{*}}{\rightarrow} Q$. We use Proposition 3.10]again to see that

$$
(\Delta ; \Theta \vdash D) \stackrel{\alpha}{\Longrightarrow}\left(\Delta, \Delta^{\prime} ; \Theta, \Theta^{\prime} \vdash D^{\prime}\right)
$$

and we now must show that $\Delta, \Delta^{\prime} ; \Theta, \Theta^{\prime} \models C^{\prime} R D^{\prime}$. To do this we use Lemma3.11 on ( $\dagger$ ) (note that $\left.Q^{\prime} \equiv v \Delta^{\prime} .\left(\delta\left\langle\Delta^{\prime}\right\rangle \| Q\right)\right)$ to see that $\left.\Delta, \Delta^{\prime},\left[\Theta \Theta, \Theta^{\prime}\right]\right] \models P \cong{ }_{p} Q$. It is also easy to check that $\mathrm{h}$ reductions are confluent with respect to all other reductions and hence preserve contextual equivalence, that is $\stackrel{\mathrm{h}}{\rightarrow}^{*} \subseteq \cong_{p}$, so we also have $\Delta, \Delta^{\prime},\left[\left[\Theta, \Theta^{\prime}\right]\right] \models\left[\left[C^{\prime}\right]\right]_{\Theta, \Theta^{\prime}} \cong_{p}\left[\left[D^{\prime}\right]\right]_{\Theta, \Theta^{\prime}}$ because $\left[\left[C^{\prime}\right]_{\Theta, \Theta^{\prime}} \stackrel{\mathrm{h}}{\rightarrow}{ }^{*} P\right.$ and $\left[\left[D^{\prime}\right]\right]_{\Theta, \Theta^{\prime}} \stackrel{\mathrm{h}}{\rightarrow} *$. This allows us to conclude $\Delta, \Delta^{\prime} ; \Theta, \Theta^{\prime} \models C^{\prime} \mathcal{R} D^{\prime}$ as required.

We must also consider transitions of the form

$$
(\Delta ; \Theta \vdash C) \stackrel{\tau}{\longrightarrow}\left(\Delta, \Delta^{\prime} ; \Theta, \Theta^{\prime} \vdash C^{\prime}\right) .
$$

These can be dealt with as above but in this case no $\mathcal{T}_{\alpha}^{\Delta}$ is needed.

Corollary 3.13 (Full abstraction). For all terms $P, Q$ of $\mathrm{HO} \pi$ :

$$
\Gamma \models P \approx^{o} Q \quad \text { if and only if } \quad \Gamma \models P \cong Q
$$

Proof. Follows from Corollary 3.8 Lemma 2.2 and the previous theorem.

\section{CONCLUding REMARKS}

We have re-examined the use of labelled transitions to characterise contextual equivalence in the higher-order $\pi$ calculus. The technique of augmenting the core syntax with extra operators to assist in the definition of the labelled transitions allows use to give a direct proof of soundness of bisimilarity for contextual equivalence. This advances Sangiorgi's analagous result by allowing recursive types also.

We believe that the technique of using extra operators to describe the points of interaction with the environment in the lts is fairly robust and should be applicable to many higher-order languages. Indeed, this was the approach that the authors developed for their work on concurrent objects [8].

We have only concerned ourselves with the characterisation of contextual equivalence in $\mathrm{HO} \pi$ and so far have not studied Sangiorgi's translation of higher-order to first-order mobility. Thus, the restriction to finite types for his translation is still necessary. It would be interesting to investigate whether the current work could be of use in removing this type restriction for his translation also. 


\section{Appendix A. Proof of The Context Lemma}

We recall the statement of Lemma2.2 and detail its proof here.

$$
\Gamma \models P \cong Q \quad \text { if and only if } \Gamma \models P \cong{ }_{p} Q .
$$

The force of this lemma is to show that the simplified form of observational testing allowed by $\cong_{p}$ is sufficient to capture the power of full contextual testing. In order to prove this we essentially need to show that $\cong_{p}$ is preserved by the operators of $\mathrm{HO} \pi$. For the most part, this can be done directly and is stated in Lemma A.1 below.

\section{Lemma A.1.}

(1) If $\Delta, x: T \models P \cong_{p} Q$ and $\Delta \vdash v: T$ then $\Delta \models(x: T) P \cdot v \cong_{p}(x: T) Q \cdot v$.

(2) If $\Delta, x: T \models P \cong_{p} Q$ and $\Delta \vdash a: \operatorname{ch}[T]$ then $\Delta \models a(x: T) P \cong_{p} a(x: T) Q$.

(3) If $\Delta \models P \cong_{p} Q, \Delta \vdash w: T$ and $\Delta \vdash a: \operatorname{ch}[T]$ then $\Delta \models a\langle w\rangle P \cong_{p} a\langle w\rangle Q$.

(4) If $\Delta \models P_{1} \cong_{p} Q_{1}$ and $\Delta \models P_{2} \cong{ }_{p} Q_{2}$ then $\Delta \models$ if $v=w$ then $P_{1}$ else $P_{2} \cong_{p}$ if $v=w$ then $Q_{1}$ else $Q_{2}$.

(5) If $\Delta, a: T \models P \cong_{p} Q$ then $\Delta \models v(a: T)$. $(P) \cong_{p} v(a: T)$. (Q).

(6) If $\Delta \models P_{1} \cong{ }_{p} Q_{1}$ and $\Delta \models P_{2} \cong{ }_{p} Q_{2}$ then $\Delta \models P_{1}\left\|P_{2} \cong_{p} Q_{1}\right\| Q_{2}$.

(7) If $\Delta \models P \cong{ }_{p} Q$ then $\Delta \models * P \cong{ }_{p} * Q$.

Proof. The majority of these are straightforward by exhibiting appropriate symmetric, reductionclosed, \|-contextual, barb-preserving relations. As an example of this we show the case for input prefixing (Case 2). We define $\mathcal{R}$ so that $\cong_{p} \subseteq \mathcal{R}$ and moreover

$$
\Delta \models a(x: T) P\|R \mathcal{R} a(x: T) Q\| R \text { for any } \Delta \vdash R
$$

It is clear that $\mathcal{R}$ is symmetric, barb-preserving and $\|$-contextual so if we can show that it is reduction-closed then we may conclude that $\mathcal{R}$ coincides with $\cong_{p}$ and we have our result.

Suppose that $(\dagger)$ holds and

$$
a(x: T) P \| R \rightarrow P^{\prime} .
$$

We know then that either $R \longrightarrow R^{\prime}$ and $P^{\prime} \equiv a(x: T) P \| R^{\prime}$ or the reduction came about by interaction, that is $R \equiv v \Delta^{\prime} .\left(a\langle v\rangle R^{\prime \prime} \| R^{\prime \prime \prime}\right)$ with $a \notin \Delta^{\prime}$ and by writing $R^{\prime}$ for $R^{\prime \prime} \| R^{\prime \prime \prime}$ we have $P^{\prime} \equiv v \Delta^{\prime} .\left(P[v / x] \| R^{\prime}\right)$ for some $\Delta, \Delta^{\prime} \vdash v$ and $\Delta, \Delta^{\prime} \vdash R^{\prime}$. If the former is true then we see immediately that

$$
a(x: T) Q\|R \rightarrow a(x: T) Q\| R^{\prime}
$$

where

$$
\Delta \models a(x: T) P\left\|R^{\prime} R a(x: T) Q\right\| R^{\prime} .
$$

If instead the latter is true then we use the fact that

$$
\Delta, x: T \models P \cong{ }_{p} Q
$$

to see that $\Delta, \Delta^{\prime} \models P[v / x] \cong_{p} Q[v / x]$ and note that

$$
a(x: T) Q \| R \rightarrow v \Delta^{\prime} .\left(Q[v / x] \| R^{\prime}\right)
$$

where (using \|-contextuality and Case 5)

$$
\Delta \models v \Delta^{\prime} .\left(P[v / x] \| R^{\prime}\right) \cong_{p} v \Delta^{\prime} .\left(Q[v / x] \| R^{\prime}\right)
$$

as required. 
Notice that there are two particular cases which are not covered by this lemma: application of a function to, and output of higher-order $\cong_{p}$-related values (c.f. Corollary A.11). Establishing that $\cong_{p}$ is preserved in these cases can be done directly but is a little more involved. We notice that the property we require in both cases follows immediately from Substitutivity (cf. Corollary A.10), that is (ignoring types):

$$
\text { if } P \cong{ }_{p} Q \text { then } R[(x) P / y] \cong{ }_{p} R[(x) Q / y] .
$$

The remainder of the appendix is devoted to achieving this. The proof follows a very similar scheme to the proof of Proposition 4.2.6 in [10] but simplified to avoid any use of induction on type as appeared there.

Lemma A.2. If $\Delta \vdash(x: T) P \cdot w$ then $\Delta \models(x: T) P \cdot w \cong_{p} P[w / x]$.

In the following we will make use of a "bisimulation up to" argument [12].

Definition A.3. A type-indexed relation $\mathcal{R}$ is reduction-closed up to $\left(=, \cong_{p}\right)$ whenever $\Delta \models P R Q$ and $P \longrightarrow P^{\prime}$ implies there exists some $Q^{\prime}$ such that $Q \Longrightarrow Q^{\prime}$ and $\Delta \models P^{\prime} \mathcal{R} \cong{ }_{p} Q^{\prime}$.

Lemma A.4. For any type-indexed relation $\mathcal{R}$ which is symmetric, reduction-closed up to $\left(=, \cong_{p}\right)$, $\|$-contextual and barb-preserving, $\mathcal{R} \subseteq \cong_{p}$.

Definition A.5. We say that $x$ is (un)guarded in $P$ whenever:

(1) if $x \notin P$ then $x$ is (un)guarded in $P$,

(2) if $x \notin w$ then $x$ is unguarded in $x \cdot w$,

(3) if $v \neq x$ then $x$ is guarded in $v \cdot w$,

(4) $x$ is guarded in $v(y: T) P, v\langle w\rangle P$, and if $v=w$ then $P$ else $Q$, and

(5) if $x$ is (un)guarded in $P$ and $Q$ then $x$ is (un)guarded in $v(a: T)$. (P), $P \| Q$ and $* P$.

Lemma A.6. For any $\Delta, y: T \rightarrow \diamond \vdash R$ with $y$ guarded in $R$ and for any $\Delta \vdash v: T \rightarrow \diamond$ and $\Delta \vdash w: T \rightarrow \diamond$, if $R[v / y] \rightarrow R^{\prime}$ then $R^{\prime}=R^{\prime \prime}[v / y]$ for some $R^{\prime \prime}$ and moreover, $R[w / y] \rightarrow R^{\prime \prime}[w / y]$.

Proof. We first observe that as $\Delta \vdash v: T \rightarrow \diamond$ it must be the case that $v$ is an abstraction and not a channel name. From this it is routine to check that the required property holds for the reduction axioms. Furthermore, if $y$ is guarded in $\mathcal{E}[P]$ then $y$ is guarded in $P$ and so the required property is preserved by reduction in evaluation contexts.

Lemma A.7. For any $P$ and $x$ we can find $Q$ and $y$ such that $x$ is guarded in $Q, y$ is unguarded in $Q$ and $P=Q[x / y]$.

Proof. A routine induction on $P$.

Lemma A.8 (Unguarded Substitutivity). If $\Delta, x: T \models P \cong_{p} Q$ and $\Delta, y: T \rightarrow \diamond \vdash R$ and $y$ is unguarded in $R$ then $\Delta \models R[(x: T) P / y] \cong{ }_{p} R[(x: T) Q / y]$.

Proof. We proceed by induction on the structure of $R$. If $y \notin R$ then the result is immediate. If $R$ is not of the form $v \cdot w$, the result follows easily by induction by making use of Lemma.1 Otherwise, since $y$ is unguarded in $R$ we must have that $R$ is of the form $y \cdot w$ with $y \notin w$. Hence:

$$
\begin{array}{rlll}
\Delta \models R[(x: T) P / y] & =(x: T) P \cdot w & & \text { (as } R=y \cdot w \text { and } y \notin w) \\
& \cong_{p} P[w / x] & & \text { (by Lemma A.2) } \\
& \cong_{p} Q[w / x] & & \text { (by hypothesis) } \\
& \cong_{p}(x: T) Q \cdot w & \text { (by Lemma A.2) } \\
& =R[(x: T) P / y] & & \text { (as } R=y \cdot w \text { and } y \notin w) .
\end{array}
$$

as required. 
Lemma A.9 (Guarded Substitutivity). If $\Delta, x: T \models P \cong{ }_{p} Q$ and $\Delta, y: T \rightarrow \diamond \vdash R$ and $y$ is guarded in $R$ then $\Delta=R[(x: T) P / y] \cong_{p} R[(x: T) Q / y]$.

Proof. Let $\mathcal{R}$ be defined as

$$
\Delta \models R^{\prime}[(x: T) P / y] \mathcal{R} R^{\prime}[(x: T) Q / y] \text { whenever } \Delta, y: T \rightarrow \diamond \vdash R^{\prime} \text { and } y \text { is guarded in } R^{\prime}
$$

We show that $\mathcal{R}$ is symmetric, reduction-closed up to $\left(=, \cong_{p}\right), \|$-contextual, and barb-preserving and so the result follows by Lemma A.4 Symmetry, \|-contextuality, and barb-preservation are direct. For reduction-closure up to $\left(=, \cong_{p}\right)$ we suppose:

$$
R^{\prime}[(x: T) P / y] \rightarrow R^{\prime \prime}
$$

By Lemma A.6 we have that $R^{\prime \prime}=R^{\prime \prime \prime}[(x: T) P / y]$ and moreover:

$$
R^{\prime}[(x: T) Q / y] \rightarrow R^{\prime \prime \prime}[(x: T) Q / y]
$$

We use Lemma A.7 to find a $R^{\prime \prime \prime \prime}$ and $z$ such that $y$ is guarded in $R^{\prime \prime \prime \prime}, z$ is unguarded in $R^{\prime \prime \prime \prime}$ and $R^{\prime \prime \prime}=R^{\prime \prime \prime \prime}[z / y]$. Hence:

$$
\begin{array}{rlll}
R^{\prime \prime} & = & R^{\prime \prime \prime}[(x: T) P / y] & \text { (from above) } \\
& =R^{\prime \prime \prime \prime}[(x: T) P / y,(x: T) P / z] & \text { (from above) } \\
\mathcal{R} & R^{\prime \prime \prime \prime \prime}[(x: T) Q / y,(x: T) P / z] & \text { (from definition of } \left.\mathcal{R} \text { and } y \text { guarded in } R^{\prime \prime \prime \prime}[(x: T) P / z]\right) \\
\cong & R^{\prime \prime \prime \prime}[(x: T) Q / y,(x: T) Q / z] & \text { (from Lemma A.8 and } \left.z \text { unguarded in } R^{\prime \prime \prime \prime}[(x: T) Q / y]\right) \\
= & R^{\prime \prime \prime}[(x: T) Q / y] & & \text { (from above) }
\end{array}
$$

as required.

Corollary A.10. If $\Delta, x: T \models P \cong_{p} Q$ and $\Delta, y: T \rightarrow \diamond \vdash R$ then $\Delta \models R[(x: T) P / y] \cong_{p} R[(x: T) Q / y]$.

Proof. Follows from Lemmas A.7 A.8 and A.9

\section{Corollary A.11.}

(1) If $\Delta, x: T \models P \cong_{p} Q$ and $\Delta \vdash v: T \rightarrow \diamond$ then $\Delta \models v \cdot(x: T) P \cong_{p} v \cdot(x: T) Q$.

(2) If $\Delta, x: T \models P \cong{ }_{p} Q, \Delta \vdash a: \operatorname{ch}[T \rightarrow \diamond]$ and $\Delta \vdash R$ then $\Delta \models a\langle(x: T) P\rangle R \cong_{p} a\langle(x: T) Q\rangle R$.

Proof. Follows from Corollary A.10

Proof of Lemma 2.2; The 'only if' direction is immediate. For the converse it is sufficient to show that $\cong_{p}$ is preserved by each process operator of $\mathrm{HO} \pi$ as demonstrated by Lemma A.1 and Corollary A.11

\section{APPENDIX B. MERGE IS A PARTIAL FUNCTION}

Proof of Proposition 3.3: We consider the rewriting relation $\rightarrow$ which we will define as the one-step rewriting used to define the merge operation:

$$
\begin{array}{rlrl}
C & \rightarrow & & \text { if } C \text { doesn't contain }\langle k \Leftarrow v\rangle \text { for any } k, v \\
v(\vec{a}: \vec{T}) .(\langle k \Leftarrow v\rangle \| C) & \rightarrow v(\vec{a}: \vec{T}) .\left(C\left[v / \tau_{k}\right]\right) & \text { if } \tau_{k} \notin v
\end{array}
$$

It is easy to see that $\rightarrow$ is a terminating rewriting relation. Moreover, the rewriting will terminate with a $\checkmark$ from $C$ (so that $\langle\langle C\rangle\rangle$ is defined) exactly when $\operatorname{rg}(C)$ is acyclic. To see this we consider the effect of $\rightarrow$ on reference graphs: for

$$
\langle k \Leftarrow v\rangle \| C \quad \rightarrow \quad C\left[v / \tau_{k}\right]
$$


the reference graph of $\langle k \Leftarrow v\rangle \| C$ has the node $k$ removed and any edges such that

$$
l^{\prime} \mapsto k \mapsto l
$$

for $l^{\prime}, l \neq k$, are replaced with an edge

$$
l^{\prime} \mapsto l
$$

all other edges involving $k$ are removed. So if node $k$ is involved in a cycle before rewriting occurs, that is

$$
l \mapsto^{*} k \mapsto^{*} l
$$

for some $l$, then either it is a tight loop, that is $l=k$ and $k \mapsto k$, or $l \neq k$ and the cycle still exist after rewriting as $l \mapsto^{*} l$. The side-condition on the rewrite rule forbids tight loops hence we see that $\rightarrow$ preserves cyclicity. That is:

if $C \rightarrow C^{\prime}$ then $\operatorname{rg}(C)$ is acyclic if and only if $\operatorname{rg}\left(C^{\prime}\right)$ is acyclic.

Now, suppose that $\langle\langle C\rangle\rangle$ is defined. We know that there exists a finite sequence

$$
C \rightarrow C_{1} \rightarrow \cdots \rightarrow C_{n} \rightarrow \checkmark
$$

with $\langle\langle C\rangle\rangle=C_{n}$. We know that $\operatorname{rg}\left(C_{n}\right)$ is acyclic as it contains no edges. Thus, $\operatorname{rg}(C)$ is acyclic also. Conversely, suppose that $\operatorname{rg}(C)$ is acyclic. Then as $\rightarrow$ is terminating there must be a finite sequence

$$
C \rightarrow C_{1} \rightarrow \cdots \rightarrow C_{n}
$$

such that $C_{n}$ cannot be rewritten. There are two possibilities for this: either $\operatorname{rg}\left(C_{n}\right)$ contains a tight loop, or $C_{n}$ is $\checkmark$. We see that $\operatorname{rg}(C)$ is acyclic, so $C_{n}$ is acyclic too and therefore cannot contain a tight loop. Thus $C_{n}$ is $\checkmark$ and $\langle\langle C\rangle\rangle$ is defined.

To show that $\langle\langle\cdot\rangle\rangle$ is a well-defined partial function it suffices to show that it is strongly confluent for acyclic terms. Note that if $v a: T .(C) \rightarrow C^{\prime}$ then either $C^{\prime}$ is $\checkmark$ or $C^{\prime} \equiv v a: T$. ( $\left.C^{\prime \prime}\right)$ such that $C \rightarrow C^{\prime \prime}$. So without loss of generality suppose that

$$
C \rightarrow C_{1} \quad \text { and } \quad C \rightarrow C_{2}
$$

for

so that

$$
C \equiv C_{1}^{\prime} \|\left\langle k_{1} \Leftarrow v_{1}\right\rangle \quad \text { and } \quad C \equiv C_{2}^{\prime} \|\left\langle k_{2} \Leftarrow v_{2}\right\rangle
$$

$$
C_{1} \equiv C_{1}^{\prime}\left[v_{1} / \tau_{k_{1}}\right] \quad \text { and } \quad C_{2} \equiv C_{2}^{\prime}\left[v_{2} / \tau_{k_{2}}\right]
$$

So either, $k_{1}=k_{2}$ in which case $C_{1} \equiv C_{2}$ or $k_{1} \neq l_{2}$ and

$$
C_{1}^{\prime} \equiv C_{3}^{\prime} \|\left\langle k_{2} \Leftarrow v_{2}\right\rangle \quad \text { and } \quad C_{2}^{\prime} \equiv C_{3}^{\prime} \|\left\langle k_{1} \Leftarrow v_{1}\right\rangle
$$

We notice that

$$
\begin{aligned}
C_{1} & \equiv C_{1}^{\prime}\left[v_{1} / \tau_{k_{1}}\right] \\
& \equiv\left(C_{3}^{\prime} \|\left\langle k_{2} \Leftarrow v_{2}\right\rangle\right)\left[v_{1} / \tau_{k_{1}}\right] \\
& \equiv C_{3}^{\prime}\left[v_{1} / \tau_{k_{1}}\right] \|\left\langle k_{2} \Leftarrow v_{2}\left[v_{1} / \tau_{k_{1}}\right]\right\rangle \\
\text { (acyclicity implies } \left.\tau_{k_{2}} \notin v_{2}\left[v_{1} / \tau_{k_{1}}\right]\right) & \rightarrow C_{3}^{\prime}\left[v_{1} / \tau_{k_{1}}\right]\left[v_{2}\left[v_{1} / \tau_{k_{1}}\right] / \tau_{k_{2}}\right] \\
& \equiv C_{3}^{\prime}\left[v_{1}\left[v_{2}\left[v_{1} / \tau_{k_{1}}\right] / \tau_{k_{2}}\right] / \tau_{k_{1}}, v_{2}\left[v_{1} / \tau_{k_{1}}\right] / \tau_{k_{2}}\right] \\
\text { (acyclicity) } & \equiv C_{3}^{\prime}\left[v_{1}\left[v_{2} / \tau_{k_{2}}\right] / \tau_{k_{1}}, v_{2}\left[v_{1} / \tau_{k_{1}}\right] / \tau_{k_{2}}\right] \\
\text { (def) } & \equiv C_{3}
\end{aligned}
$$

By a symmetric argument we see that $C_{2} \rightarrow C_{3}^{\prime}\left[v_{2}\left[v_{1} / \tau_{k_{1}}\right] / \tau_{k_{2}}, v_{1}\left[v_{2} / \tau_{k_{2}}\right] / \tau_{k_{1}}\right]$ and, by definition, this is just $C_{3}$ so we have $C_{2} \rightarrow C_{3}$. Thus $\rightarrow$ is strongly confluent for acyclic terms and hence $\langle\langle\cdot\rangle\rangle$ is well-defined. 


\section{REFERENCES}

[1] L. Cardelli and A. Gordon. Mobile ambients. In Proc. Foundations of Software Science and Computation Structures (FoSSaCS), Lecture Notes in Computer Science. Springer-Verlag, 1998.

[2] C. Fournet and G. Gonthier. A hierarchy of equivalences for asynchronous calculi. In Proc. Int. Conf. Automata, Languages and Programming (ICALP), volume 1443 of Lecture Notes in Computer Science. Springer-Verlag, 1998.

[3] C. Fournet, G. Gonthier, J-J. Levy, L. Maranget, and D. Remy. A calculus of mobile agents. In Proc. CONCUR, volume 1119 of Lecture Notes in Computer Science. Springer-Verlag, 1996.

[4] A. Giacalone, P. Mishra, and S. Prasad. Facile: A symmetric integration of concurrent and functional programming. In Proc. TAPSOFT, volume 352 of Lecture Notes in Computer Science, pages 184-209. Springer-Verlag, 1989.

[5] M. Hennessy and J. Rathke. Typed behavioural equivalences for processes in the presence of subtyping. In Proc. Computing: the Australasian Theory Symposium (CATS), Electronic Notes in Theoretical Computer Science. Elsevier, 2002.

[6] K. Honda and N. Yoshida. On reduction-based process semantics. Theoretical Computer Science, 152(2):437-486, 1995.

[7] A.S.A Jeffrey and J. Rathke. A theory of bisimulation for a fragment of Concurrent ML with local names. In Proc. IEEE Symp. Logic in Computer Science (LICS), pages 311-321. Computer Society Press, 2000.

[8] A.S.A Jeffrey and J. Rathke. A fully abstract may testing semantics for concurrent objects. In Proc. IEEE Symp. Logic in Computer Science (LICS), pages 101-112. Computer Society Press, 2002.

[9] J. Riely and M. Hennessy. A typed language for distributed mobile processes. In Proc. ACM Conf. Principles of Programming Languages (POPL). ACM Press, 1998.

[10] D. Sangiorgi. Expressing Mobility in Process Algebras: First-Order and Higher-Order Paradigms. PhD thesis, University of Edinburgh, 1993.

[11] D. Sangiorgi. Bisimulation for higher-order process calculi. Information and Computation, 131(2):141-178, 1996.

[12] D. Sangiorgi and R. Milner. On the problem of 'weak bisimulation up to'. In Proc. CONCUR, volume 630 of Lecture Notes in Computer Science, pages 32-46. Springer-Verlag, 1992.

[13] D. Sangiorgi and D. Walker. The pi-calculus: A Theory of mobile processes. Cambridge University Press, 2001.

[14] B. Thomsen. Calculi for Higher-Order Communicating Systems. PhD thesis, University of London, 1990.

[15] J. Vitek and G. Castagna. Seal: A framework for secure mobile computations. In Internet Programming Languages, volume 1686 of Lecture Notes in Computer Science. Springer-Verlag, 1999.

This work is licensed under the Creative Commons Attribution-NoDerivs License. To view a copy of this license, visit http://creativecommons.org/licenses/by-nd/2.0/ or send a letter to Creative Commons, 559 Nathan Abbott Way, Stanford, California 94305, USA. 